Bundesgesundheitsbl 2018 · 61:239-248 https://doi.org/10.1007/s00103-017-2682-y

@ U Umweltbundesamt, Dessau-Roßlau 2018

Empfehlungen des Arbeitskreises Lüftung (AK Lüftung) am Umweltbundesamt

\title{
Anforderungen an Lüftungskonzeptionen in Gebäuden - Teil I: Bildungseinrichtungen
}

Dipl.-Ing. Heidemarie Schütz, BBSR, Ref. II 5 - Nachhaltiges Bauen, Berlin Jun. Prof. PhD Dirk Schwede, Universität Stuttgart, Stuttgart

Dipl.-Ing. Jens Schuberth, Umweltbundesamt, FG I 2.4, Dessau-Roßlau

Dipl.-Ing. Peter Tappler, Arbeitskreis Innenraumluft am BMLFUW (Umweltministerium), Wien

Prof. Dr.med. Gerhard A. Wiesmüller, Gesundheitsamt Stadt Köln

\section{Kernbotschaften}

1. Die Konzentration von Kohlendioxid $\left(\mathrm{CO}_{2}\right)$ in der Innenraumluft von Unterrichtsräumen darf im Mittel einer Unterrichtseinheit eine Konzentration von 1000 ppm nicht überschreiten.

2. Eine Lüftung über Fenster allein reicht zum Erreichen einer guten Innenraumluftqualität während des Unterrichts in Schulgebäuden nicht aus. Eine Konzeption bestehend aus Grundlüftung über mechanische Lüftungsanlagen und Zusatzlüftungsmöglichkeit über Fenster in den Pausen (hybride Lüftung) wird vom AK Lüftung dringend empfohlen.

3. Für jedes Unterrichtsgebäude (Schulen, Kitas, Universitäten) ist ein Lüftungskonzept zu erstellen, das sowohl Aspekte für die Planung und Ausführung von Neubauten und Sanierungsarbeiten im Bestand als auch für den täglichen Betrieb umfasst. Lüftungskonzepte sind raumweise - unabhängig von der Lüftungsart (mechanische Lüftung oder über Fenster) - für den Sommer- und den Winterbetrieb getrennt zu erstellen.
4. Lüftung muss funktional, bedarfsgerecht und wirtschaftlich (Investition und Betrieb) sein. Die Planung hierzu ist zwischen den beteiligten Fachplanern und -ingenieuren, Bauherrn und Nutzern abzustimmen.

5. Die Lüftungssysteme sollen über Wärme- und Feuchterückgewinnung verfügen und bedarfsgerecht regelbar sein. Neben der $\mathrm{CO}_{2}$-Konzentration stellt die relative Feuchte der Luft ein weiteres Kriterium für gute bzw. angemessene Innenraumluftqualität dar. Sie soll vorzugsweise im Bereich zwischen 30-60\% liegen.

6. Eine sorgfältige Inbetriebnahme ist Voraussetzung für den Betrieb. Den Nutzern ist eine Einweisung in das jeweilige Lüftungskonzept mit klar verständlichen schriftlichen Handlungsempfehlungen zu übergeben.

7. In Schulbestandsbauten, die nicht sogleich mit einer Lüftungstechnik versehen werden können, ist auch während einer Unterrichtseinheit eine Lüftung über die Fenster unbedingt erforderlich. Die Verwendung eines $\mathrm{CO}_{2}$-Sensors (Lüftungsampel) kann Hilfestellung geben, um eine Verbesserung der Fensterlüftung zu erreichen.

8. In der Betriebsphase sind vorhandene technische Anlagen regelmäßig zu überprüfen und zu reinigen, um ihre ordnungsgemäße Funktionsfähigkeit zu gewährleisten. Dazu gehört auch der Nachweis auf die Einhaltung des o.g. $\mathrm{CO}_{2}$-Leitwertes unter realen Nutzungsbedingungen. 


\section{Einleitung}

Wir halten uns in Mitteleuropa den weitaus überwiegenden Teil des Tages in geschlossenen Räumen auf, zu Hause, in Bahnen, Bussen, Pkw oder am Arbeitsplatz. Gute Innenraumluftqualität ist daher für unser Wohlbefinden und unsere Gesundheit unerlässlich. Die Innenraumluftgüte wird neben baulichen Einflüssen wesentlich durch das Verhalten der Nutzer bestimmt, unter anderem durch das Einbringen von Stoffen durch Inventar und Reinigungsmittel sowie das Heiz- und Lüftungsverhalten. Moderne Heizungsanlagen nehmen den Nutzern weitgehend die individuelle Regelungsnotwendigkeit beim Heizen ab. Beim Lüften ist es anders. Mittlerweile gibt es zwar in Deutschland und anderen Ländern der EU ausgereifte und in der Praxis vielfach erprobte technische Lüftungseinrichtungen, die dem Verbraucher/Nutzer das „aktive" und eigenverantwortliche Lüften über die Fenster abnehmen oder dies zumindest ergänzen. Die Verbreitung war lange Zeit vor allem auf größere Büro- und Verwaltungsgebäude sowie auf spezielle Einrichtungen mit hohem Anspruch an die Raumluftgüte beschränkt. Erst in den letzten Jahren setzt sich Lüftungstechnik zunehmend auch in weiteren Bereichen wie dem Wohnungsbau durch.

Lüften ist aufgrund der langen Aufenthaltszeiten im Innenraum und der vielfältig eingebrachten chemischen und biologischen Stoffe, gleich ob bei Bestandsgebäuden oder Neubauten, mehr denn je wichtig, um eine gute Raumluftqualität aufrechtzuerhalten-häufig sogar, um eine gute Raumluftqualität überhaupt erst zu erreichen.

Das gilt maßgeblich auch für die Innenraumluft in Bildungseinrichtungen wie Schulen, Hochschulen oder Universitäten, aber auch für Kindertagesstätten. In Bildungseinrichtungen erfordert die Lüftungssituation besondere Aufmerksamkeit. Die vergleichsweise große Zahl an Personen auf oft engem Raum macht schon allein aufgrund des vom Menschen ausgeatmeten Kohlendioxids ein regelmäßiges Lüften unerlässlich, um die gesundheitlich-hygienischen Vorgaben der Innenraumluftgüte zu erreichen. Bloßes Lüften-ab und an-über die Fenster reicht dann nicht mehr aus. Neben Stoffparametern wie Kohlendioxid $\left(\mathrm{CO}_{2}\right)$ und anderen chemischen Stoffen sind die kognitive Leistungsfähigkeit und die Behaglichkeit während des Unterrichts für Schüler und Lehrer ein wichtiges Kriterium, um den Lernerfolg zu unterstützen und Unwohlsein oder gar Ausfall durch Erkrankungen, die durch den Aufenthalt im Innenraum bedingt sind, vorzubeugen.

Lüftungsplanung in Unterrichtsgebäuden ist eine integrale Aufgabe, die bereits in der Vorentwurfsphase beginnt, die gesamte Bauausführung begleitet und ein umfassendes Betriebs- und Wartungskonzept erfordert. Sie umfasst zahlreiche interdisziplinäre Aspekte. Dazu gehören neben der Innenraumlufthygiene u.a. die Behaglichkeit, Raumluftfeuchte, Praktikabilität und Energieeffizienz. Sowohl im Neubau als auch im sanierten und unsanierten Altbau zeigen Messungen, dass Fensterlüftung bei den gegenwärtigen Randbedingungen hinsichtlich der Personenbelegung und Raumgrößen in Bildungseinrichtungen allein nicht geeignet ist, während der Nutzungszeiten gute Innenraumluftqualitäten sowie ein gutes und behagliches Innenraumklima zu gewährleisten. Das Umweltbundesamt hat dem bereits 2009 in seinem Leitfaden für die Innenraumlufthygiene in Schulgebäuden (www.umweltbundesamt.de) Rechnung getragen und empfiehlt bei Neubau von Unterrichtsgebäuden den Einbau technischer Lüftungssysteme. Die $\mathrm{CO}_{2}$-Konzentration in der Innenraumluft von Klassenräumen stellt dabei den Leitwert für die Beurteilung dar.

Voraussetzungen für eine gute Innenraumluftqualität in Bildungseinrichtungen sind:

\section{- niedriger $\mathrm{CO}_{2}$-Gehalt der Innen- Zielvorgaben} raumluft,

- Behaglichkeit bezüglich Raumlufttemperatur, relativer Luftfeuchte, Luftbewegung (Zugluftfreiheit, Luftschichtung) und Schallpegel,

- Verwendung emissionsarmer Bauprodukte und Einrichtungsgegenstände zur Verringerung des Eintrags chemischer Stoffe,

- geringer Gehalt nutzer- und nutzungsbedingter Luftverunreinigungen,
- Bedienfreundlichkeit lüftungstechnischer Einrichtungen und Beachtung der Fehlertoleranz im Betrieb,

- einfache und kostengünstige regelmäßige technische und hygienische Wartung von Lüftungs- und Heizungsanlagen,

- robuste, zuverlässige und wenig störanfällige Anlagen,

- Einflussmöglichkeiten und Pflichten der Nutzenden.

Die genannten Aspekte sind als Zielvorgaben in den Planungsprozess bei Sanierung und Neubau von Bildungseinrichtungen einzubeziehen. Sie stellen eine Grundlage für das Erreichen einer angemessenen Innenraumluftqualität dar und zeigen die hohen Anforderungen auf, die bei der Lüftungsplanung bedacht werden müssen.

Zur Einhaltung einer hygienisch unbedenklichen Innenraumluft ist gemäß Ausschuss für Innenraumrichtwerte (AIR) beim Umweltbundesamt ein gesundheitlich-hygienischer Leitwert von 1000 ppm $\mathrm{CO}_{2}$ einzuhalten. Dies sichert gleichzeitig die Aufrechterhaltung leistungsfördernder Umgebungsbedingungen beim Lernen.

Hinsichtlich der Anforderungen an die Lüftung gemäß Arbeitsstättenverordnung (ArbStättV), konkretisiert mit der ASR A3.6, dient ebenfalls die Einhaltung einer $\mathrm{CO}_{2}$-Konzentration von $1000 \mathrm{ppm}$ als Maß zur Einhaltung des Schutzzieles einer "gesundheitlich zuträglichen Atemluft". Gemäß ASR A3.6 sind raumlufttechnische Anlagen (RLT) erforderlich, wenn eine freie Lüftung bei Einhaltung der dort beschriebenen Lüftungsquerschnitte nicht ausreicht.

Zunächst gilt es, die grundlegenden Zielvorgaben hinsichtlich der Innenraumluftqualität miteinander zu kombinieren und zu optimieren. Sie dienen als Richtschnur für die Planung und Nutzung und müssen bei jedem individuellen Projekt auf möglichst sinnvolle und effiziente Weise zusammengeführt werden. 


\section{Kohlendioxidkonzentration in der Raumluft}

Die Kohlendioxid- $\left(\mathrm{CO}_{2}-\right)$ Konzentration in der Raumluft ist das zentrale Kriterium für gute bzw. angemessene Innenraumluftqualität in Unterrichtsräumen. Die Einhaltung definierter Ziel- bzw. Maximalwerte der $\mathrm{CO}_{2}$-Konzentration kann darüber hinaus als Indikator für die Einhaltung weiterer Luftqualitätsrichtwerte, z.B. für flüchtige organische Verbindungen (VOC) und andere Stoffe, angesehen werden. Für alle Arten von Bildungseinrichtungen gilt als Richtwert eine $\mathrm{CO}_{2}$ Konzentration der Innenraumluft als Mittelwert von 1000 ppm über die Dauer einer Nutzungseinheit (in Schulen i.d.R. eine Schulunterrichtsstunde, an Universitäten eine Vorlesungseinheit). Kurzzeitig erhöhte $\mathrm{CO}_{2}$-Momentanwerte von z.B. 1500 ppm sind akzeptabel, wenn der Mittelwert von $1000 \mathrm{ppm} \mathrm{CO}_{2}$ während der Unterrichtseinheit eingehalten wird.

\section{Relative Feuchte}

Neben der $\mathrm{CO}_{2}$-Konzentration stellt die relative Feuchte der Luft ein weiteres Kriterium für gute bzw. angemessene Innenraumluftqualität dar. Die relative Raumluftfeuchte sollte idealerweise im Bereich zwischen 30-60\% liegen (allgemeiner Behaglichkeitsbereich). Im Winter sind kurzzeitige Unterschreitungen bis auf etwa 20$25 \%$ möglich. Weitere Unterschreitungen (unter 20\%) werden mit vermehrten Reizerscheinungen in Verbindung gebracht, dauerhaft erhöhte Werte (oberhalb von $60 \%$ ) erhöhen das Schimmelrisiko.

\section{Zugluftfreiheit}

Die Luftgeschwindigkeit soll im genutzten Unterrichtsraum einen Wert von $0,15 \mathrm{~m} / \mathrm{s}$ nicht überschreiten. Die Zuluft der Lüftungsanlage sollte eine Temperatur von mindestens $17^{\circ} \mathrm{C}$ aufweisen. Die Temperaturdifferenz von einströmender Luft zur Raumluft beträgt im optimalen Fall $2 \mathrm{~K}$ (Kelvin) bis max. 4 K. Diese Anforderungen an Arbeits- bzw. übliche Aufenthaltsräume können allein mittels Fensterlüftung oder mit lediglich mechanischen Abluftanlagen bei winterlichen Außentemperaturen in der Regel nicht erreicht werden.
Hygieneanforderungen und

Wartung von Lüftungsanlagen

Die Anlagen sind so auszuführen, dass die Innenraumlufthygiene nicht beeinträchtigt wird. Insbesondere müssen die Bereiche der Außenluftansaugung und der Lüftungsleitungen über Revisionsöffnungen zugänglich und ohne hohen Aufwand zu reinigen sein. Bei Planung und Montage gilt es, den Hygieneanforderungen besondere Sorgfalt zukommen zu lassen. Ein regelmäßiger Filterwechsel muss auf einfache Art und Weise sichergestellt sein.

Um einen technisch und hygienisch einwandfreien Betrieb der Anlage sicherzustellen und somit negativen gesundheitlichen Auswirkungen vorzubeugen, sind bei Inbetriebnahme eine technische und hygienische Abnahme sowie regelmäßige technische und hygienische Inspektionen, Wartungen und Instandsetzungen notwendig. Die zeitlichen Intervalle hängen vom Anlagentyp ab (vgl. u.a. VDI Reihe 6022-siehe Abschnitt Normen).

\section{Schallschutz}

Lüftungsanlagen ermöglichen es, Fenster geschlossen zu halten und insbesondere in Gebäuden an Hauptverkehrsstraßen, in Flughafennähe oder bei sonstigen äußeren Lärmquellen einen guten Schutz gegenüber Außenlärm zu erzielen. Andererseits verursachen auch mechanische Lüftungsanlagen aufgrund ihrer Wirkungsweise Geräusche, die in den Aufenthalts- bzw. Unterrichtsräumen begrenzt werden müssen. Messbar sind diese als Schalldruckpegel im Raum. Richtwerte für den Auslegungsschalldruckpegel liegen z.B. für Unterrichts-, Fach-, Mehrzweckräume und Lehrerzimmer bei maximal $35 \mathrm{~dB}(\mathrm{~A})$, bei Sporthallen bei maximal $40 \mathrm{~dB}(\mathrm{~A})$. Gegebenenfalls können auch höhere Anforderungen erforderlich werden.

\section{Bewahrung der}

Eigenverantwortlichkeit und hoher Komfort für die Nutzer

Zahlreiche Untersuchungen zeigen im Ergebnis immer wieder, dass die Möglich- keit zur persönlichen Einflussnahme auf das Innenraumklima, wie Fenster öffnen oder Temperaturregelung, von entscheidender Bedeutung für das Wohlbefinden und die Zufriedenheit der Nutzer in Aufenthaltsräumen ist. Zentral gesteuerte Lüftungsanlagen ohne diese Einflussmöglichkeiten erhöhen die Anonymität und können zu Unwohlsein führen. Die integrale Betrachtung physiologischer und psychologischer Einflüsse auf die Befindlichkeit des Menschen ist daher wichtig für angenehmes Lernen und Arbeiten in Unterrichtsgebäuden. Dabei sind also nicht nur Qualität und Quantität von Raumklimafaktoren, sondern auch die Interaktion zwischen Nutzer, räumlichen Gegebenheiten und gebäudetechnischer Ausstattung von Bedeutung.

Die verschiedenen Nutzergruppen in Bildungseinrichtungen sollen die Möglichkeit erhalten, aktiv Einfluss nehmen zu können. Hierbei kommen hybride Lüftungskonzepte dem Bedürfnis nach eigenständigem Handeln und Wohlbefinden am besten entgegen. Dabei wird die Grundlüftung über eine mechanische Lüftungsanlage durchgeführt; zusätzlich kann über Fensterlüftung eine individuelle $\mathrm{Zu}$ satzlüftung geschehen. Beides zusammen sorgt für eine angemessene Innenraumluftqualität und vom Nutzer akzeptierte Lüftungsform. Damit solche Konzepte in der Praxis funktionieren, müssen Lehrkräfte und Schüler in die entsprechenden Lüftungsintervalle und -zeiten eingewiesen werden. Eine sensorgesteuerte $\mathrm{CO}_{2}-$ Ampel, die es bereits preiswert gibt, zeigt an, wann die $\mathrm{CO}_{2}$-Konzentrationen in einen kritischen Bereich kommen, und unterstützt das eigenverantwortliche Handeln der Nutzenden.

\section{Planungsparameter}

Generell soll bei allen Neubau- und Sanierungsprojekten, aber auch für bestehende Schulgebäude ohne anstehende Sanierungen immer ein Lüftungskonzept (vgl. - Infobox 1) für alle Lüftungsarten, für den Winter und Sommer getrennt erarbeitet werden.

Die Anforderung, einen ausreichenden Luftwechsel zu gewährleisten, ist dabei Grundvoraussetzung, um hygienische Innenraumluftverhältnisse erreichen $\mathrm{zu}$ 


\section{Infobox 1 Lüftungskonzept}

Mit dem Begriff, Lüftungskonzept“ ist im Folgenden ein anwenderorientiertes Konzept gemeint. Dieses ist unter Beteiligung der relevanten Fachplaner, der Bauherrnvertreter und der Nutzer aufzustellen, um die erforderliche Abstimmung mit dem Sicherheits- und Unfallverhütungskonzept, dem Energiekonzept, dem Akustikkonzept, dem Brandschutzkonzept und dem Nutzungskonzept zu erzielen. Es beschreibt für jeden Raum:

- die Anforderung an die Innenraumlufthygiene (hier insbesondere $\mathrm{CO}_{2}$-Konzentration),

- die Rahmenbedingungen,

- die Festlegung der erforderlichen lüftungstechnischen Maßnahmen und

- die Auswahl der Lüftungsart für die einzelnen Räume.

Mit dem Lüftungskonzept ist die Praxistauglichkeit der Lüftung für die einzelnen Räume rechnerisch nachzuweisen. Für gleichartige Räume kann dieser Nachweis an einem repräsentativen Raum geführt werden. Das Lüftungskonzept soll bereits in einer frühen Planungsphase erstellt und planungsbegleitend verwendet werden. Zudem ist es auf den jeweiligen Planungsstand fortzuschreiben. Das Konzept beinhaltet keine detaillierte Bemessung und Berechnung von lüftungstechnischen Anlagen, sondern lediglich die aus hygienischer Sicht erforderlichen Parameter und Angaben, um:

- eine ausreichende Be- und Entlüftung zu gewährleisten und diese nachvollziehbar prüfen zu können,

- den Nutzern eine Handreichung zur praktischen Umsetzung des Konzeptes (z. B. Regelbarkeit einer mechanischen Lüftungsanlage, ggf. erforderliche Lüftungsintervalle über Fensteröffnungen) zu geben,

- Hinweise zur Wartung und Instandhaltung für den Betrieb einer raumlufttechnischen Anlage zu dokumentieren,

- als Basis für eine Erfolgskontrolle (Monitoring) in der Nutzungsphase herangezogen werden zu können.

\section{Allgemeine Parameter}

Da im Fokus des anwendungsorientierten Lüftungskonzeptes die raumweise Betrachtung steht, sind darin-für jede Lüftungsart-folgende Angaben zu dokumentieren:

- der erforderliche Außenluftvolumenstrom pro Raum,

- die jeweilige Lüftungsart (mechanische Lüftung und/oder in Kombination mit Fensterlüftung),

- die Anzahl der Räume mit den entsprechenden Raumabmessungen,

- die jeweilige Personenanzahl und Altersstufe, für die die Räume konzipiert sind,

- die geplanten täglichen Unterrichts- und Pausenzeiten,

- die Zeit und Dauer der Luftzufuhr,

- die $\mathrm{CO}_{2}$ - Außenluftkonzentration am Standort,

- der Nachweis der Einhaltung der Anforderungen an die $\mathrm{CO}_{2}$-Konzentration bspw. mithilfe des $\mathrm{CO}_{2}$-Onlinemodells des Niedersächsischen Landesgesundheitsamtes (https://www.nlga.niedersachsen.de/umweltmedizin/luft/projekt_lufthygiene_schulen/co2modellsoftware/co2-modellsoftware-19316.html).

\section{Einsatz der Fensterlüftung}

Ist für Aufenthaltsräume eine Fensterlüftung oder eine unterstützende Fensterlüftung geplant (hybrides Lüftungskonzept), ist der vorgesehene personenbezogene Außenluftvolumenstrom pro Raum sowohl für den Sommer- als auch für den Winterfall durch Berechnung nachzuweisen. Für die Berechnung können die VDI 6040 (siehe Rechentool der VDI 6040) oder die DIN 15242 herangezogen werden.

Für die Dokumentation der Berechnungsergebnisse sind - zusätzlich zu den im Abschnitt „Allgemeine Parameter" aufgeführten Punkten - alle weiteren erforderlichen Planungsparameter anzugeben. Insbesondere zählen hierzu:

- die Anzahl der öffenbaren Fensterflügel mit den geplanten und für die Nutzung geeigneten Öffnungsweiten,

- die Lüftungsintervalle (z. B. 1 $\times$ nach $45 \mathrm{~min}$ ),

- die entsprechende Lüftungsdauer,

- die angenommene Temperaturdifferenz innen/außen für den Sommer- und den Winterfall,

- die mittlere Windgeschwindigkeit am Standort sowie

- Angaben zur Organisation der Verantwortlichkeit zur Fensteröffnung.

Weiterhin ist darzustellen, ob es sich um eine einseitige Fensterlüftung oder um eine Querlüftung handelt.

\section{Handlungsempfehlungen für den Nutzer}

Insbesondere für Räume, denen ein hybrides Lüftungskonzept zugrunde liegt, müssen die Raumnutzer über die Funktionsweise und das Zusammenspiel der einzelnen Komponenten informiert werden. Dazu zählen beispielsweise:

- der Anteil der Grundlüftung über die mechanische Lüftungsanlage ggf. mit den Regelungsmöglichkeiten,

- die erforderlichen Maßnahmen für die unterstützende freie Lüftung über die Fenster mit den geplanten Lüftungszeiten und -intervallen und

- die Funktionsweise und Handhabung eines etwaigen $\mathrm{CO}_{2}$-Sensors.

können. Lüftungsanlagen sollen so ausgeführt und betrieben werden, dass die relative Feuchte der Innenraumluft bei kaltem und trockenem Außenklima im Winter nicht zu niedrig wird (vgl. Zielvorgaben). Trockene Innenraumluft wird oft mit Lüftungstechnik in Verbindung gebracht, ist jedoch kein anlagenspezifisches Problem, sondern tritt ebenso auf, wenn bei intensiver Fensterlüftung ein ausreichend hoher Luftwechsel erreicht werden soll.

Vor anlagentechnischen Auslegungen und Berechnungen sind bauliche und organisatorische Möglichkeiten (z. B. Raumvolumen, Fensterdimensionierung, Klassenstärke) zu prüfen. So sollten beim Einsatz einer mechanischen Lüftungsanlage alle Aufenthaltsbereiche (ausgenommen innen liegende Räume) über zu öffnende Fenster verfügen, um die Möglichkeit der Fensterlüftung weitgehend auszunutzen und um beim Ausfall der Anlage (gilt auch bei kombinierten Lüftungskonzepten) hygienisch annähernd zuträglich Innenraumluftverhältnisse gewährleisten zu können. Außerhalb der Heizperiode sollte Fensterlüftung zudem so oft wie möglich genutzt werden. 


\section{Infobox 2 Lüftungstechnik energieeffizient und nutzerfreundlich}

Ein hohes interdisziplinäres Fachwissen ist erforderlich, um Lüftungskonzepte (vgl. • Infobox 1) fachgerecht zu realisieren. Der ausgeführte Wärmerückgewinnungsgrad und die spezifische Ventilatorleistung der Anlagentechnik wirken sich auf den Wärme- und Stromverbrauch und damit auf die Energiekosten im Betrieb aus. Der Wärmebereitstellungsgrad der Anlagen sollte deshalb mindestens im Bereich von 80-85\% liegen. Die Ventilatorleistung inkl. Regelung der Anlage sollte unter $0,3 \mathrm{Wh} / \mathrm{m}^{3}$ betragen. Es sollten Filter der Energieklasse A oder B nach EN 779-2012 (EuroventZertifizierung) zum Einsatz kommen. Ineffiziente Lüftungsgeräte dürfen seit 2016 nicht mehr in der EU in Verkehr gebracht werden; ab 2018 gelten noch strengere Effizienzanforderungen und es muss eine Filterwechselanzeige vorhanden sein (Verordnung 2014/1253/EU).

\section{Einsparung von Heizenergie}

Durch Lüftungsanlagen mit Wärmerückgewinnung werden die Lüftungswärmeverluste gegenüber Fensterlüftung um rechnerisch $40-50 \mathrm{kWh} /\left(\mathrm{m}^{2}\right.$ a) gesenkt. In der Realität liegen die Werte oft niedriger, weil der Luftwechsel ohne Lüftungsanlage meist geringer ist bei gleichzeitig schlechterer Innenraumluftqualität. Neben einer hochwertigen Gebäudehülle stellt die Wärmerückgewinnung den zweiten wichtigen Aspekt zukunftsorientierter Gebäudestandards dar. Als Ergebnis kann ein Heizwärmebedarf von $15 \mathrm{kWh} /\left(\mathrm{m}^{2} \mathrm{a}\right)$ im Bereich des Passivhaus-, Null- oder Plusenergiestandards mit guter Luftqualität erreicht werden, wie mittlerweile zahlreiche gebaute Gebäude zeigen.

\section{Luftdichtheit des Gebäudes}

Beim Einsatz von Lüftungsanlagen ist ein Dichtheitskonzept für die Gebäudehülle von zentraler Bedeutung, weil erwärmte Luft und auch Feuchte über Leckagen verloren gehen, statt über den Wärmetauscher geleitet zu werden, um die Energie auf die einströmende Außenluft zu übertragen. Als Zielwert der Luftdichtheitsmessung gilt $n_{50} \leq 0,6 \mathrm{~h}^{-1}$.

\section{Bedienfreundlichkeit}

Zur Bedienfreundlichkeit und bezüglich Toleranz gegenüber Fehlern im Betrieb gilt: Die Gebäudetechnik sollte inkl. Regelung so einfach wie möglich ausgeführt werden und von den Nutzern nachvollziehbar sein, Funktion und Bedienung sollten jeder Lehrende und die Schüler intuitiv verstehen können. Die Standardwartung inkl. Filterwechsel muss mit geringem Aufwand von einem Hausmeister leistbar sein.

\section{Investitionskosten}

Die Kosten zahlreicher ausgeführter Lüftungsanlagen sind teilweise erheblich. Mittlerweile kann jedoch von Planern verlangt werden, eine hochwertige Lüftungsanlage mit Wärme- und Feuchterückgewinnung für etwa $10.000-15.000 €$ pro Klassenraum ausführen zu lassen. Bei einer Kosten-Nutzen-Betrachtung muss neben Effizienzgründen vor allem die Erzielung guter innenraumlufthygienischer Bedingungen angeführt werden, Faktoren, die monetär schwer bewertet werden können. Die Planungen für solche Anlagen müssen mit der nötigen Fachkompetenz, Sorgfalt und Sparsamkeitsprinzipien im Sinne des Nutzers/Bauherren/Investors erfolgen.

\section{Neubau und Sanierung}

Bereits im Vorentwurfsstadium eines Neubaus oder einer Schulsanierung soll eine integrale Planung mit den beteiligten Fachingenieuren und Nutzern durchgeführt werden. Innenraumlufthygiene, Emissionsverhalten der Baustoffe, Brandschutz, Schallschutz sowie funktionale Anforderungen müssen miteinander und zueinander optimiert werden. Sowohl beim Neubau als auch bei umfänglichen Sanierungen ist grundsätzlich der Einbau von lüftungstechnischen Systemen mit Wärme- und Feuchterückgewinnung zu fordern. Als gesundheitlich-hygienische Anforderung für die planerische Auslegung gilt eine $\mathrm{CO}_{2}-$ Konzentration der Innenraumluft als arithmetischer Mittelwert von 1000 ppm über die Dauer einer Nutzungseinheit (siehe Zielvorgaben). Die $\mathrm{CO}_{2}$-Momentanwerte können dabei, wie Erfahrungswerte und Rechenmodelle zeigen, bis auf etwa 1500 ppm steigen. Kurzzeitige $\mathrm{CO}_{2}$ Spitzen sind weder ein Planungsfehler noch hygienisch ein Risiko, solange der $\mathrm{CO}_{2}$-Mittelwert über die Unterrichtsstunde eingehalten wird.

Die zentrale Planungsaufgabe besteht darin, die Lüftungsanlage mit ihren Komponenten bedarfsgerecht und möglichst wirtschaftlich $\mathrm{zu}$ dimensionieren. Neben dem Außenluftvolumenstrom haben auch das Lüftungssystem, die Lufteinbringung und -absaugung (z.B. Misch- oder Quellluft), die Art der Luftauslässe, die Größe des Lüftungskanalnetzes und des Lüftungsgerätes sowie die Mess- und Regelungstechnik Einfluss auf das Ergebnis der Luftqualität und auch auf die Investitions- und Betriebskosten.

In Ergänzung zur üblichen Pausenlüftung wird als Richtwert für die Auslegung von zentralen und dezentralen Lüftungsanlagen eine Geräteauslegung auf einen Außenvolumenstrom von etwa $25 \mathrm{~m}^{3} / \mathrm{h}$ pro Person empfohlen. Das Ziel des Erreichens der 1000 ppm $\mathrm{CO}_{2}$ im Mittel über die Unterrichtseinheit ist bei der rechnerischen Auslegung jedoch unbedingt zu beachten. Dies ist als mittlere Berechnungsgröße anzusehen, die im Einzelfall und bei besonderen Situationen (siehe unten) nach oben und unten entsprechend variieren kann.

Abweichungen können sich u.a. durch das Alter und die Aktivitäten der Raumnutzer, aufgrund örtlicher Bedingungen (z.B. Verkehr, Lärm) sowie durch besondere Nutzungsarten (z.B. Kindertagesstätten) ergeben. In Kitas wird man in der Regel mit niedrigeren Außenluftvolumenströmen auskommen, während man z.B. für Räume von Oberstufen und für Erwachsene oder, falls eine Pausenlüftung aufgrund von Umgebungsbedingungen nicht möglich sein sollte, höhere Außenluftvolumenströme benötigt.

So wie eine Unterdimensionierung des Außenluftvolumenstromes aus hygienisch-gesundheitlicher Sicht zu vermeiden ist, ist eine Überdimensionierung schon aus Gründen erhöhter Investitions- und Betriebskosten zu vermeiden (vgl. - Infobox 2). Praktische Beispiele zeigen, dass durch optimierte Planung des Lüftungskonzepts der $\mathrm{CO}_{2}$-Zielwertkorridor unter Vermeidung einer Überdimensionierung der Lüftungsanlagen auch mit geringerem Außenluftvolumenstrom eingehalten werden kann. Das Erreichen der Zielvorgaben ist planerisch und rechnerisch in jedem Einzelfall $\mathrm{zu}$ belegen (vgl. auch Berechnungsbeispiele in - Infobox 6).

Zur Vermeidung einer Überdimensionierung von Lüftungsanlagen sind z. B. folgende Aspekte von Bedeutung:

- Durch großzügige Lüftungsquerschnitte der Fenster kann der Pausenlüftungsanteil erhöht werden, dazu gehört eine wirksame und sichere Anordnung der zu öffnenden Fensterflächen, im Idealfall mit Querlüftung. 


\section{Infobox 3 Lüftungstechnik und sommerlicher Wärmeschutz}

Zum Wohlbefinden in Innenräumen gehört nicht nur eine Komforttemperatur im Winter, sondern auch im Sommer gilt es, einer übermäßigen Überhitzung der Räume entgegenzutreten. Grundsätzlich ist es nicht Aufgabe von Lüftungsanlagen, den sommerlichen Wärmeschutz zu gewährleisten. In erster Linie muss dies durch bauliche Lösungen erzielt werden. Das sind vor allem:

- eine außen liegende Verschattung der Fenster (mit Rolläden, vorstehenden Gebäudeteilen oder Pflanzen), die verhindert, dass einfallendes Sonnenlicht die Räume aufheizt,

- guter Wärmeschutz an Wänden und Fenstern, die den Wärmeeintrag über die Außenflächen verringern, und

- eine hohe Bauteilmasse, die tagsüber eintretende Wärmelasten „abpuffern“ kann, die nachts mittels Lüftung wieder nach draußen abgeführt werden.

Lüftungsanlagen können jedoch flankierend helfen und über das Konzept der Nachtlüftung in den sommerlichen Wärmeschutz eingebunden werden. Eine einfache Lösung mit geringem

Energiebedarf ist z. B. die Ansaugung der kühlen Außenluft über Fenster/Nachtlüftungsöffnungen

(ggf. gesteuert motorisch zu öffnen) in den Klassenräumen und die Abführung der Abluft über die Lüftungsanlage (Abluftschaltung).

Nicht empfohlen werden Erdreichwärmetauscher aufgrund des geringen Kühleffekts in Verbindung mit hohem Investitionsaufwand. Zudem besteht bei Luft-Erdreich-Wärmetauschern die Gefahr mikrobieller Belastungen.

Lüftungsgeräte mit Wärmerückgewinnung müssen seit 2016 einen „Sommerbypass“ haben, der den Wärmetauscher umgeht, sodass nachts die Raumluft gegen kühle Außenluft ausgetauscht werden kann (Verordnung 2014/1253/EU).

- Größere Raumhöhe bzw. größeres Raumvolumen von z. B. über $7,5 \mathrm{~m}^{3}$ pro Person können helfen, die Lüftungssituation zu verbessern, indem der Zeitpunkt der Lüftungsnotwendigkeit dadurch um einige Minuten nach hinten verschoben werden kann.

- Realistische Annahmen zu Raumbelegungen und Berücksichtigung der Gleichzeitigkeitsfaktoren (Reduzierung der rechnerischen Gesamtluftmenge aufgrund der nicht gleichzeitig möglichen Belegung aller belüfteten Räume mit maximaler Belegungsstärke). Der Ansatz der Gleichzeitigkeit ist auch abhängig von der Art der raumweisen Luftmengenregelung.

- Berücksichtigung der $\mathrm{CO}_{2}$-Abgabe in Abhängigkeit vom Alter der Nutzer,

- Berechnungsnachweis der Außenluftvolumenströme und der zu erwartenden $\mathrm{CO}_{2}$-Belastung inkl. Beitrag der Fensterlüftung (vgl. - Infobox 1 und 4 weiter unten).

Eine erhöhte Auslegung kann erforderlich sein bei folgenden Aspekten:

- keine Möglichkeit zur Hybridlüftung über Fenster (z. B. an lärmbelasteten Standorten),

- eingeschränkte Lüftungsquerschnitte der Fenster,

- höhere $\mathrm{CO}_{2}$-Abgabe bei erwachsenen Schülern und erhöhter Aktivität.
Dezentrale Lüftungsanlagen sollten so ausgelegt werden, dass sie aus Gründen des Schallschutzes und der Energieeffizienz bei $80 \%$ ihrer Nennleistung betrieben werden. Bei zentralen Lüftungssystemen ist das Thema der Gleichzeitigkeitsfaktoren (siehe oben) und der Annahmen zur Personenbelegung von zentraler Bedeutung. Es ist Aufgabe der qualifizierten und verantwortlichen Planer, mittels realistischer Festlegung der Planungsparameter und ausgewogener Berücksichtigung der zahlreichen Planungsaspekte eine möglichst wirtschaftliche Anlage zu planen. Dazu ist eine intensive Abstimmung mit den Bauherren und späteren Nutzern inkl. der Lehrer erforderlich. Auch den Nutzern sollte ein Wissen um die sinnvoll abgewogenen Grenzen des Lüftungssystems vermittelt werden, um die spätere Akzeptanz und eine bedarfsgerechte Nutzung (unter Einbeziehung der Nutzer) zu erhöhen. Bereits in der Planungsphase sollte auch die Betreuung der technischen Anlagen nach der Fertigstellung und in der Nutzungsphase sowie die Erfolgskontrolle des Lüftungskonzeptes unter Praxisbedingungen geregelt werden. Für die Nutzer ist eine Einweisung in das Lüftungskonzept (vgl. • Infobox 1) mit entsprechenden Handlungsempfehlungen zu erstellen.

Die Lüftungsregelung soll möglichst einfach und bedarfsgerecht sein. Bei Ein- zelgeräten (dezentrale Geräte) können Zeit-, Präsenz- oder auch $\mathrm{CO}_{2}$-Regelungen realisiert werden. Das Gleiche gilt für zentrale Anlagen, bei denen die Funktionalität sowie der Aufwand für Investitions- und Betriebskosten dieser Regelparameter in der Planung unbedingt berücksichtigt werden müssen.

Lüftungsanlagen sollten zusätzlich zur Wärme- auch mit Feuchterückgewinnung geplant werden, um zu niedrige Luftfeuchte in der kalten Jahreszeit zu vermeiden. Im Sommer gilt es, einer zu starken Gebäudeaufheizung entgegen zu wirken (vgl. - Infobox 3). Der Einsatz von Luftfiltern ist bei mechanischen Lüftungsanlagen zwingend erforderlich und richtet sich bzgl. der Qualität nach den Anforderungen im Innenraum und den Außenluftbedingungen, es sind die Mindestvorgaben der entsprechenden Lüftungsnormen zu beachten. Die Einhaltung von Austausch-/Wartungszyklen für die Luftfilter beeinflusst maßgeblich die Zuluft- und auch die Innenraumluftqualität. Eine optimierte Wärmerückgewinnung, eine Bedarfsregelung und eine geringe spezifische Ventilatorleistung der Anlagentechnik senken den Wärme- und Stromverbrauch und wirken sich damit positiv auf die Gesamtenergiekosten im Betrieb aus.

Bei Gebäudesanierungen gilt: Fenstertausch inkl. Einbau von z. B. motorisch zu öffnenden Fensterflügeln stellt meist keine gering investive Maßnahme dar. $\mathrm{Zu}$ dem muss solch ein Austausch unbedingt im Rahmen eines baulichen Gesamtkonzepts durchgeführt werden, welches Dämmmaßnahmen in der Fassade in die Überlegungen einbezieht. Dies gilt vor allem dann, wenn die bestehenden Außenwände eine relativ schlechte Dämmwirkung haben und zu befürchten ist, dass mit dem Einbau neuer Fenster die Gefahr der Tauwasserbildung auf den Wandflächen bzw. in Wandecken oder Laibungen mit Folge von Schimmelbildung entsteht. Aus Kostengründen ist es oftmals naheliegender, dann dezentrale Lüftungsanlagen einzubauen. Ihr Vorteil liegt darin, dass sie auch sukzessive raumweise eingebaut werden können. Im Zuge der energetischen Sanierung können später auch die Fenster erneuert und die Wärmedämmung verbessert werden. 
Infobox 4 Sachgerechtes Lüften in Bestandsbauten mit Fensterlüftung (ohne Lüftungstechnik)

Bei Schulgebäuden im Bestand, die noch nicht über lüftungstechnische Einrichtungen verfügen, muss unbedingt regelmäßig vor und während des Unterrichts gelüftet werden. Es wird ausdrücklich darauf hingewiesen, dass die folgenden Empfehlungen keine Alternative zur zukünftigen Verwendung mechanischer Lüftungen in Kombination mit manuellem Lüften über Fenster (Hybridlüftung) darstellen. Es handelt sich um Maßnahmen, die im Regelfall nicht dazu führen, dass die geforderten $\mathrm{CO}_{2}$-Werte (1000 ppm im Unterrichtsstundenmittel) eingehalten werden, jedoch im Rahmen der Lüftungsmöglichkeiten der meisten Bestandsbauten die Innenraumluftqualität in Unterrichtsgebäuden so gut wie gegenwärtig möglich halten sollen.

Wichtig ist, dass beim Lüften alle Fenster regelmäßig-auch in der Heizperiode-kurzzeitig weit geöffnet werden (Stoßlüftung). Ein Lüften zum Flur hin ist ebenso unzweckmäßig wie ein Lüften über Kippstellung der Fenster (Ausnahme: dauerhaft gekippte Fenster im Sommer).

Die Stoßlüftung soll erfolgen:

- Vor Unterrichtsbeginn sollen alle Fenster in einem Klassenraum 5-10 min so weit wie möglich geöffnet werden. Das gilt auch und besonders nach Wochenenden und wenn die Räume längere Zeit nicht genutzt wurden.

- In jeder kurzen Pause sollen alle Fenster im Klassenraum ebenfalls so weit wie möglich für die gesamte Dauer der Unterrichtspause weit geöffnet werden. Die Heizkörperthermostaten können dabei während der Heizperiode heruntergedreht werden.

- In jeder langen Pause (Hofpause) soll die Lüftungsdauer vorzugsweise ebenfalls über die ganze Pause erfolgen. Im Winter bei sehr niedrigen Temperaturen unter dem Gefrierpunkt reicht es, die Fenster in der langen Pause ca. 5-10 min geöffnet zu halten. Vorhandene Heizkörperthermostaten können zuvor gedrosselt werden.

- Während des Unterrichts muss bei üblicher Raumbelegung zusätzlich gelüftet werden. Eine generelle Vorgabe gibt es hier nicht. Eine $\mathrm{CO}_{2}$-Ampel kann helfen, die notwendigen Lüftungsintervalle während des Unterrichts anzuzeigen. Eine zusätzliche „Kipplüftung“ kann während des Unterrichts den Luftaustausch unterstützen, sofern keine unzumutbaren Bedingungen hinsichtlich Temperatur und Zugerscheinungen sowie durch Lärm oder Einwirkung von Immissionen von außen entstehen. Im Sommer können die Fenster auch dauerhaft auf Kippstellung bleiben, sofern Lärmaspekte oder zusätzliche Raumaufheizungen von außen dem nicht entgegenstehen.

Bestehen Sicherheitsbedenken gegen ein Lüften, weil z. B. kleine Kinder während der Pausen in den Klassenräumen verbleiben und man Sorge hat, dass Kinder aus dem Fenster stürzen könnten, muss das Lüften dennoch erfolgen, dann jedoch unter Anwesenheit einer Aufsicht o. Ä. Alternativ müssen die Kinder den Raum während der Pause verlassen. Es gelten die gängigen Unfallverhütungsvorschriften.

\section{Infobox 5 Untersuchungsergebnisse}

Um die Vorgaben für verschiedene Lüftungsszenarien und -empfehlungen zu überprüfen, fanden im Zeitraum von Oktober 2016 bis Januar 2017 in sieben Städten in Deutschland Messungen der $\mathrm{CO}_{2}$-Raumluftkonzentrationen in verschiedenen Schultypen, wie Grund-, Real-, Berufsschulen, Gymnasien etc., mit unterschiedlichen baulichen und anlagentechnischen Rahmenbedingungen statt. Diese reichten von neu gebauten Schulen mit mechanischen Lüftungsanlagen, über sanierte Schulen mit dezentralen Lüftungsanlagen, hybriden Lüftungssystemen bzw. motorisch gesteuerter Fensterlüftung sowie unsanierten Schulen mit ausschließlicher Fensterlüftung, teils unterstützt durch Lüftungsampeln. Insgesamt wurde die Raumluftqualität anhand der $\mathrm{CO}_{2}$-Bilanzen in 35 Unterrichtsräumen gemessen. Die Auswertungen zeigen, dass alleinige manuelle Fensterlüftung nicht ausreichend ist, den gesundheitlich-hygienischen Leitwert der $\mathrm{CO}_{2}$-Raumluftkonzentration (1000 ppm) zu gewährleisten.

Eine ausführliche Darstellung der Ergebnisse erfolgt erst nach Drucklegung dieser Empfehlungen und wird unter anderem in der Schriftenreihe des Vereins für Wasser-, Boden- und Lufthygiene im Verlauf des Sommers 2018 erscheinen.

\section{Empfehlungen für \\ Kindertagesstätten}

Der Begriff Kindertagesstätten (Kitas) umfasst in der Regel Kinderkrippen (0-3 Jahre), Kindertagesstätten (2-7 Jahre), Kinderhorte (6-10/12 Jahre) sowie auch Kinder- und Jugendhäuser (etwa 7-17 Jahre).
Auch für Kitas gelten die beschriebenen Anforderungen an eine gute Innenraumluftqualität. Bei Neubau- oder umfassenden Sanierungsmaßnahmen gelten die gleichen Ziele für die $\mathrm{CO}_{2}$-Konzentration, die relative Luftfeuchte, die Zugluftfreiheit sowie den Schalldruckpegel und die zu öffnenden Fensterflächen, analog zu Schulgebäuden und Universitäten. Aufgrund der anderen Nutzungsstruktur und Randbedingungen gegenüber klassischen Schulgebäuden, den meist offenen Raumnutzungen, den meist jüngeren Kindern, dem starken Innen-Außen-Bezug, den häufig schwankenden Raumbelegungen bei meist offenen Türen und der generell individuelleren Betreuung stellt sich die $\mathrm{CO}_{2}$-Problematik nicht in der Brisanz wie bei klassischen Schulgebäuden. Begründete Abweichungen zu den beschriebenen Auslegungsaußenluftvolumenströmen sind möglich und die dargestellten Randbedingungen zu beachten. Dezentrale Lüftungsgeräte für die Hauptaufenthaltsbereiche können beispielsweise mit einfachen Zeitsteuerungen betrieben und bei Bedarf im Sommer zur wirksamen Nachtabkühlung verwendet werden.

\section{Bestandsgebäude}

Stehen in Bestandsgebäuden keine oder keine sofortigen Investitionsmittel für eine mechanisch betriebene Lüftung zur Verfügung, muss zunächst weiterhin ein Luftaustausch mittels Fensterlüftung erfolgen. Dieser muss dann gezielt und sachgerecht erfolgen, um eine größtmögliche Annäherung an die Luftqualitätsvorgaben für $\mathrm{CO}_{2} \mathrm{zu}$ erreichen (vgl. - Infobox 4). Grundlage dafür ist eine fundierte Information, wann eine Fensterlüftung erfolgen muss (vgl. - Infobox 4 und 5). Dies sollte durch eine gezielte Aufklärung geschehen; auch Lüftungsampeln können hilfreiche Informationen liefern. In der wärmeren Jahreszeit können Fenster dauerhaft geöffnet werden. Ausnahmen gelten z. B bei erforderlichem Lärmschutz oder in Zeiten sehr hoher Immission von Abgasen und Stäuben, die von außen in die Unterrichtsräume gelangen. Bei niedrigen $\mathrm{Au}$ ßentemperaturen können dauerhaft geöffnete Fenster keine Lösung sein, weil für die Schülerinnen und Schüler eine thermische Unbehaglichkeit durch einströmende Kaltluft entsteht. Die einzige Möglichkeit besteht dann im Stoßlüften in den Pausen und während des Unterrichts. Mithilfe der Lüftungsampel zeigt sich sehr schnell, wie oft und wie lange gelüftet werden muss. In Abhängigkeit 


\section{Infobox 6 Lüftung im Klassenraum, Berechnungsbeispiele}

Wie verschiedene Lüftungsmöglichkeiten sich unmittelbar auf die $\mathrm{CO}_{2}$-Konzentrationen in Unterrichtsräumen auswirken, zeigen die folgenden Beispiele. Ohne Lüftung steigt die $\mathrm{CO}_{2}$-Konzentration im betrachteten Beispielraum innerhalb einer 45-minütigen Unterrichtseinheit um etwa 60 ppm/Person. Bei den niedrigen Jahrgangsstufen ist dieser Anstieg etwas geringer, bei den höheren Jahrgangsstufen entsprechend stärker. Am Ende der Unterrichtseinheit ergibt sich somit, selbst bei besten Ausgangsbedingungen (Außenluftkonzentration etwa 400 ppm), eine $\mathrm{CO}_{2}$-Konzentration deutlich über 2000 ppm. Mit üblichen $\mathrm{CO}_{2}$-Konzentrationen zu Beginn des Unterrichts werden sogar Werte von etwa 3000 ppm erreicht. Dies verdeutlicht die Notwendigkeit einer Lüftung während des Unterrichts.

Das Lüften während des Unterrichts kann grundsätzlich kontinuierlich (mechanische Lüftung) oder punktuell (Stoßlüftung über Fenster) erfolgen. Da punktuelles Lüften immer eine Störung des Unterrichts darstellt, wird in der Praxis im Regelfall kontinuierlich gelüftet. Die kontinuierliche Lüftung sorgt für eine stetige Verdünnung der $\mathrm{CO}_{2}$-Emissionen und wirkt damit dem Anstieg der $\mathrm{CO}_{2}$-Konzentration entgegen. Für eine Begrenzung der $\mathrm{CO}_{2}$-Konzentration auf $1000 \mathrm{ppm}$ ist im betrachteten Fall ein Volumenstrom von mindestens $30 \mathrm{~m} /$ /(h.Person) erforderlich. Für eine gemittelte $\mathrm{CO}_{2}$-Konzentration von 1000 ppm in der Unterrichtseinheit genügt eine kontinuierliche Lüftung mit ca. $25 \mathrm{~m}^{3} /(\mathrm{h} \cdot$ Person), wenn diese in der Pause durch eine Stoßlüftung über die Fenster ergänzt wird (hybride Lüftung).

Da Fensterlüftung auf der temperatur-, feuchte- und windbedingten Druckdifferenz zwischen Innen- und Außenraum basiert, ist sie stark von den klimatischen Bedingungen abhängig. Der Luftaustausch wird zudem durch die baulichen Gegebenheiten, bspw. Fenstergeometrie, beeinflusst. Bei einseitiger Stoßlüftung eines Raumes während einer Unterrichtspause (5-10 min) kann ein Volumenstrom realisiert werden, der einem Luftwechsel von 1,0-2,0 h-1 entspricht. Die $\mathrm{CO}_{2}$-Konzentration im Raum kann so, abhängig vom Ausgangswert, um bis zu $70 \%$ gesenkt werden, sofern während der Lüftung keine Personen im Unterrichtsraum anwesend sind. Für ein Absinken der $\mathrm{CO}_{2}$-Konzentration im Raum auf das Niveau der Außenluft sind längere Lüftungszeiten bzw. eine höhere Lüftungseffektivität, bspw. durch Querlüftung, erforderlich.

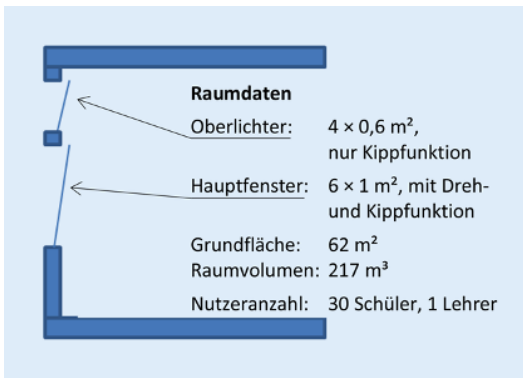

\ Vertikalschnitt Beispielraum nach VDI 6040

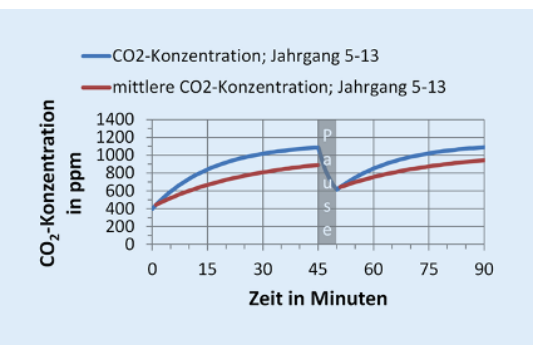

$\Delta \mathrm{CO}_{2}$-Konzentration bei hybrider Lüftung ( $25 \mathrm{~m}^{3} /(\mathrm{h} \cdot$ Person) + Stoßlüftung in der Pause)

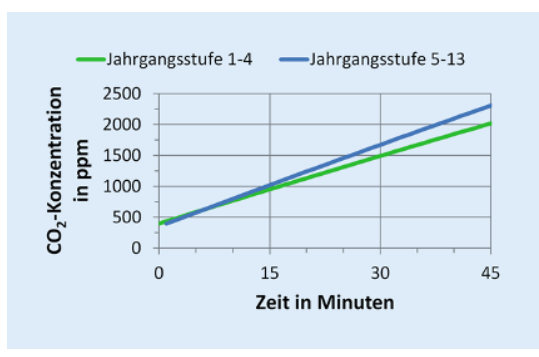

$\Delta \mathrm{CO}_{2}$-Konzentration ohne Lüftung

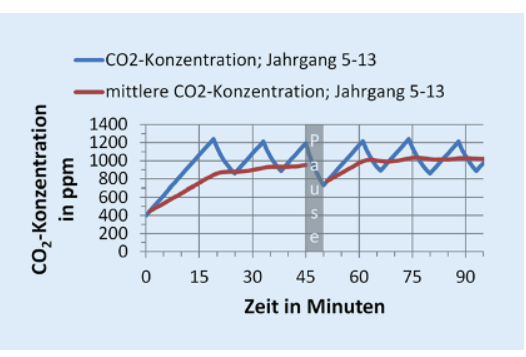

$\Delta \mathrm{CO}_{2}$-Konzentration bei punktueller Lüftung (einseitige, mehrmalige Stoßlüftung während der Unterrichtseinheiten und in der Pause)

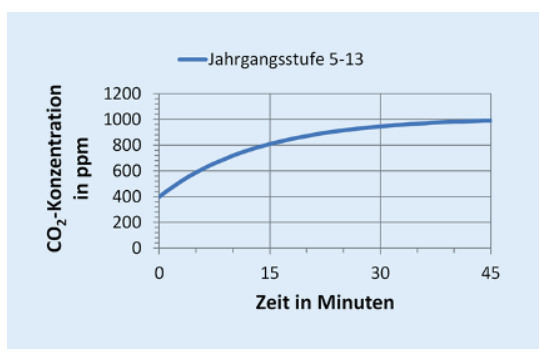

$\Delta \mathrm{CO}_{2}$-Konzentration bei kontinuierlicher Lüftung $\left(30 \mathrm{~m}^{3} /(\right.$ h.Person $\left.)\right)$ von Klassenstärke (die sich in der Praxis kaum reduzieren lässt, um bereits darüber eine Verbesserung der $\mathrm{CO}_{2}$-Bilanzen zu erreichen), Raumgröße (ebenfalls nicht veränderbar), Witterung sowie Gestaltung der Fensteröffnungen werden zusätzlich zur Pausenlüftung mehrere Lüftungsvorgänge innerhalb einer Unterrichtsstunde nötig sein (vgl. Berechnungsbeispiele, $\bullet$ Infobox 6 und 7 ). Erfahrungsgemäß ist dies in der Praxis schwer umsetzbar, weil die Akzeptanz dafür fehlt oder weil man es schlichtweg vergisst. Diese Aufgabe muss daher in gemeinsamer Verantwortung und mit klar geregelter Zuständigkeit vom Lehrkörper mithilfe der Schülerinnen und Schüler übernommen werden.

\section{Verordnungen und Normen}

Gegenwärtig existieren verschiedenartige Richtlinien, Verordnungen, technische Normen- und Regelungswerke zum Thema Lüftung bzw. Raumlufttechnik. Wesentliche, auch für Bildungseinrich- tungen relevante Planungsgrundlagen sind u. a.:

Technische Regel für ArbeitsstättenLüftung-ASR A3.6 (in der jeweils gültigen Fassung):

Technische Regeln für Arbeitsstätten (ASR) geben den Stand der Technik, Arbeitsmedizin und Arbeitshygiene sowie sonstige gesicherte arbeitswissenschaftliche Erkenntnisse für das Einrichten und Betreiben von Arbeitsstätten wieder. Die ASR A3.6 konkretisiert die Anforderungen der Verordnung über Arbeitsstätten an die Lüftung. 


\section{Infobox 7 Rechentools zur Planungsunterstützung von Lüftung in Schulen}

Bezüglich der planerischen Umsetzung von Konzepten zur mechanischen Belüftung von Innenräumen existieren diverse softwaretechnische Instrumente (Tools). Anders sieht es im Bereich der natürlichen Lüftung aus. Die hier verfügbaren wenigen Rechentools fokussieren sich jeweils nur auf Teilaspekte und betrachten das Zusammenspiel aus Raumgeometrie, Raumbelegung, Luftwechselrate, thermischem Komfort und $\mathrm{CO}_{2}$-Konzentration nicht ganzheitlich. Für eine umfassende Betrachtung der raumrelevanten Aspekte müssen daher mehrere Rechentools miteinander verschaltet werden, beispielsweise das Rechentool zur VDI 6040 und das $\mathrm{CO}_{2}$-Onlinemodell des Niedersächsischen Landesgesundheitsamtes (http://www.co2-modell.niga.niedersachsen.de/). Um Planern und Entscheidern eine verlässliche Information zur raumlufthygienischen Wirkung eines vorgesehenen Lüftungs- bzw. Raumkonzeptes zu geben, erarbeitet das Bundesinstitut für Bau-, Stadt- und Raumforschung (BBSR) derzeit ein vereinfachtes Rechenwerkzeug, das für die ganzheitliche planerische Betrachtung von Konzepten sowohl der reinen natürlichen als auch der hybriden Belüftung genutzt werden kann.

Das Rechenwerkzeug des BBSR soll anhand weniger Eingabewerte zur Raumgeometrie, zur Raumbelegung und zur vorgesehenen Lüftungsstrategie die sich einstellenden Luftwechselraten und hierauf basierend die relevanten Parameter hinsichtlich des thermischen Komforts und der $\mathrm{CO}_{2}$-Konzentration (einschließlich des zeitlichen Verlaufs) ermitteln. Anhand dieser Werte sind Vergleiche unterschiedlicher Lüftungsstrategien schnell und einfach durchzuführen. Perspektivisch ist das Rechenwerkzeug auch als Nachweisinstrument im Rahmen der Nachhaltigkeitsbewertung von Gebäuden gemäß dem BNB-Bewertungssystem (www.bnb-nachhaltigesbauen.de) vorgesehen. Weitere Informationen sind direkt beim BBSR und den anderen genannten Institutionen erhältlich.

Verordnung über energiesparenden Wärmeschutz und energiesparende Anlagentechnik bei Gebäuden (Energieeinsparverordnung-EnEV) vom 24.07 (BGBl. I S. 1519), die zuletzt durch Artikel 3 der Verordnung vom 24.15 (BGBl. I S. 1789) geändert worden ist: Zweck der Verordnung ist die Einsparung von Energie in Gebäuden. Es sind u. a. Forderungen nach luftdichten Gebäudehüllen bei Sicherstellung der zum Zwecke der Gesundheit und Beheizung erforderlichen Mindestluftwechsel sowie nach energieeffizienten Lüftungsanlagen enthalten. Es gilt die jeweils aktuelle Fassung.

DIN EN 13779-2007-Lüftung von Nichtwohngebäuden-Allgemeine Grundlagen und Anforderungen für Lüftungsund Klimaanlagen und Raumkühlsysteme: Diese europäische Norm gilt für die Planung und Ausführung von Lüftungsund Klimaanlagen in Nichtwohngebäuden, die für den Aufenthalt von Menschen bestimmt sind. Hier ist eine Kategorisierung von Luftqualitätsklassen enthalten, die von IDA 1 bis IDA 4 (IDA 1: hohe, IDA 2: mittlere, IDA 3: mäßige, IDA 4: niedrige Raumluftqualität) reichen und zu Planungsbeginn mit dem Nutzer/Bauherrn als Zielwerte zu vereinbaren sind. Die Klassifizierung sollte stets dem Typ und der vorgesehenen Nutzung des Gebäudes entsprechen. Im Wesentlichen wird dabei als zentraler Einflussfaktor auf die Raumluftqualität die Abhängigkeit von der gewünschten $\mathrm{CO}_{2}$-Raumluftkonzentration mit einer Auslegung des zu realisierenden Luftwechsels durch die Anlagentechnik festgelegt.

DIN EN 15251-2012-Eingangsparameter für das Raumklima zur Auslegung und Bewertung der Energieeffizienz von Gebäuden: Diese Norm gilt in erster Linie für nichtindustrielle Gebäude (auch Schulgebäude), bei denen die Kriterien für das Innenraumklima durch die menschliche Nutzung bestimmt werden. Hier wird in Kategorien I bis III (I: hohes, II: normales und III: annehmbares Maß an Erwartungen) wiederum in Abhängigkeit von der $\mathrm{CO}_{2}$-Raumluftkonzentration unterteilt und die Kategorie II für neue und renovierte Gebäude empfohlen.

DIN EN 16798-2015 (Entwurf)-Energieeffizienz von Gebäuden: Diese Normenreihe soll die DIN EN 13779 und DIN EN 15251 ablösen und liegt derzeit als Entwurf vor. Die einzelnen Teile befinden sich in der Gremienabstimmung; eine Veröffentlichung stand zum Zeitpunkt der Drucklegung dieser Empfehlungen noch aus.

VDI 6040 Blatt 1, Juni 2011-Raumlufttechnik-Schulen-Anforderungen: Hier sind Dimensionierungshinweise, vor allem für Luftmengen enthalten.

VDI 6040, Blatt 2, September 2015Ausführungshinweise: In der Richtlinie werden neben freier und maschineller Lüftung auch Varianten hybrider Lüftung aufgezeigt. Ausgehend von den Anforderungen an den Schulraum (Blatt 1) werden Anwendungsbeispiele (Blatt 2) dargestellt, mit denen die gestellten Anforderungen einhaltbar sind. Dazu werden personenbezogene Luftvolumenströme zur $\mathrm{Di}$ mensionierung in Abhängigkeit von Jahrgangsstufe und Aktivität angegeben sowie die Auslegungsgrundsätze für freie und maschinelle Lüftung erläutert. Es werden Vor- bzw. Nachteile der angegebenen Beispiele betrachtet. Die Richtlinie berücksichtigt ebenfalls die Forderungen von DIN EN 15251 und DIN EN ISO 7730. VDI 6022 Blatt 1-Raumlufttechnik, Raumluftqualität-Hygieneanforderungen an Raumlufttechnische Anlagen und Geräte (VDI-Lüftungsregeln): Die Richtlinie beschreibt Anforderungen an Planung, Ausführung und Betrieb von raumlufttechnischen Anlagen und Geräten aus hygienischer Sicht. Zur Sicherstellung der Qualität werden neben einer hygienischen Abnahmeuntersuchung regelmäßige Kontrollen und Inspektionen mit notwendigem Leistungsumfang festgelegt. Zusätzliche Anforderungen werden an die Qualifikation des Personals, auch des Betriebspersonals gestellt (VDI 6022 Blatt 4).

\section{Zusammenfassung der wesentlichen Aspekte}

Die Qualität der Innenraumluft in Bildungseinrichtungen ist ein wichtiger Faktor zur Sicherstellung des Gesundheitsschutzes für Lernende und Lehrende sowie aller weiteren Nutzer, die sich einen großen Teil des Tages in den Räumen von Bildungseinrichtungen aufhalten. Gleichzeitig sind die Anforderungen energiesparenden und nachhaltigen Bauens zu erfüllen. Zur Sicherstellung einer gesundheitlichen Unbedenklichkeit der Innenraumluft in sanierten und neu gebauten Schulen ist neben der Verwendung emissionsarmer Bauprodukte und Einrichtungsgegenstände ein ausreichender Luftwechsel zur Abfuhr belasteter und Zufuhr frischer Luft zwingend erforderlich.

Der Arbeitskreis Lüftung geht davon aus, dass die eingangs genannten Schutzziele des Ausschusses für Innenraumrichtwerte und der Arbeitsstättenverordnung eingehalten werden, wenn der arithmetische Mittelwert von $1000 \mathrm{ppm} \mathrm{CO}_{2}$ über 
die Dauer einer Unterrichtseinheit (in Schulen üblicherweise 45 min, ohne Pausen) nicht überschritten wird.

Nach dem aktuellen Stand von Wissenschaft und Technik und bestätigt durch zahlreiche Messungen und Simulationsberechnungen ist die alleinige Fensterlüftung nicht in der Lage, diese Anforderungen ganzjährig unter Gewährleistung einer angemessenen Temperatur und ohne Zuglufterscheinungen zu erfüllen.

Zur Gewährleistung des Gesundheitsschutzes ist es aus Sicht des Arbeitskreises daher notwendig, neben optimal gestalteten Fenstern, eine zusätzliche bedarfsgeregelte technische Lüftung einzuplanen. Dies geschieht über ein detailliertes Lüftungskonzept, welches in die Planung, aber auch in die spätere Nutzung einzubeziehen ist. Es wird empfohlen, die erforderliche personenbezogene Lüftungsrate als sogenannte Hybride Lüftung (also als Kombination von mechanischer Grund- lüftung und bedarfsweiser Zusatzlüftung über Fenster) auszuführen. Erfahrungen aus der Praxis haben gezeigt, dass die Möglichkeit der eigenständigen Fensteröffnung einen wesentlichen Anteil an der Akzeptanz und Zufriedenheit der Nutzer hat. Gleichzeitig wird es damit möglich, die Auslegung von Lüftungsanlagen um den durch die freie Lüftung gelieferten (Zusatz-)Außenluftvolumenstrom zu verringern.

Wesentlich für den erfolgreichen Betrieb sanierter oder neuer Bildungsgebäude sind außerdem die Einbeziehung der Raumnutzerinnen und Raumnutzer bereits bei der Planung der Maßnahmen, die Erfolgskontrolle des Lüftungskonzeptes unter Praxisbedingungen und die professionelle Betreuung und Wartung der technischen Anlagen nach der Fertigstellung sowie in der Nutzungsphase.

Diese Lüftungsanforderungen orientieren sich an den vorliegenden wissen- schaftlichen Erkenntnissen zu den negativen Auswirkungen unzureichender Lüftung für die Nutzer von Bildungseinrichtungen und den gesundheitlichhygienischen Anforderungen an die Innenraumluftqualität. Sowohl die Sicherstellung der kognitiven Leistungsfähigkeit und des Wohlbefindens der Nutzer als auch insbesondere die Gewährleistung der gesundheitlichen Unbedenklichkeit dürfen aus Kostengründen nicht gefährdet werden. Die Empfehlungen enthalten daher keine Kostenrechnung anhand Investitions- und Betriebskosten einerseits und Energieeinsparungen andererseits. Sie berücksichtigen jedoch Aspekte wie Kosten und Nachhaltigkeit durch Beschränkung der technischen Lüftung auf das notwendige Maß durch passgenaue Dimensionierung und die Einbeziehung der unterstützenden manuellen Fensterlüftung in die Konzeption. 